\title{
Molecular Mechanism of Species-Dependent Sweet Taste toward Artificial Sweeteners
}

\author{
Bo Liu, ${ }^{1,2}$ Matthew Ha, ${ }^{1}$ Xuan-Yu Meng, ${ }^{1}$ Tanno Kaur, ${ }^{1}$ Mohammed Khaleduzzaman, ${ }^{1}$ Zhe Zhang, ${ }^{1}$ Peihua Jiang, ${ }^{3}$ \\ $\mathrm{Xia} \mathrm{Li}{ }^{3}$ and Meng Cui ${ }^{1}$ \\ ${ }^{1}$ Department of Physiology and Biophysics, Virginia Commonwealth University, Richmond, Virginia 23298, 22College of Food and Bioengineering, Shandong \\ Institute of Light Industry, Jinan 250353, China, and ${ }^{3}$ Monell Chemical Senses Center, Philadelphia, Pennsylvania 19104
}

The heterodimer of Tas1R2 and Tas1R3 is a broadly acting sweet taste receptor, which mediates mammalian sweet taste toward natural and artificial sweeteners and sweet-tasting proteins. Perception of sweet taste is a species-selective physiological process. For instance, artificial sweeteners aspartame and neotame taste sweet to humans, apes, and Old World monkeys but not to New World monkeys and rodents. Although specific regions determining the activation of the receptors by these sweeteners have been identified, the molecular mechanism of species-dependent sweet taste remains elusive. Using human/squirrel monkey chimeras, mutagenesis, and molecular modeling, we reveal that the different responses of mammalian species toward the artificial sweeteners aspartame and neotame are determined by the steric effect of a combination of a few residues in the ligand binding pocket. Residues S40 and D142 in the human Tas1R2, which correspond to residues T40 and E142 in the squirrel monkey Tas1R2, were found to be the critical residues for the species-dependent difference in sweet taste. In addition, human Tas1R2 residue I67, which corresponds to S67 in squirrel monkey receptor, modulates the higher affinity of neotame than of aspartame. Our studies not only shed light on the molecular mechanism of speciesdependent sweet taste toward artificial sweeteners, but also provide guidance for designing novel effective artificial sweet compounds.

\section{Introduction}

Taste qualities can be categorized as sweet, bitter, sour, salty, and umami. To date, it has been well described that the family $\mathrm{C}$ G-protein-coupled receptor (GPCR) Tas1R2 and Tas1R3 heterodimer mediates the primary sensory transmission process of sweet taste perception, which can be stimulated by many diverse natural or synthetic sweet ligands, and sweet-tasting proteins (Nelson et al., 2001; Jiang et al., 2004). Each subunit of the heterodimeric receptor has a large $\mathrm{N}$-terminal extracellular domain, comprised of a Venus flytrap module (VFTM) and a small cysteine-rich domain (CRD), followed by a transmembrane domain (TMD) and an intracellular region (see Fig. $1 B$ ). Understanding how the sweet taste receptor functions at the molecular level is important for designing novel sweeteners, for example, low-caloric sweeteners with a pleasant taste (Zhang et al., 2010).

Previous studies using sweet receptor chimeras, mutagenesis, and molecular modeling showed that there are multiple potential

\footnotetext{
Received Feb. 13, 2011; revised May 17, 2011; accepted June 14, 2011.

Author contributions: B.L., Z.Z., and M.C. designed research; B.L., M.H., X.-Y.M., T.K., M.K., and M.C. performed research; B.L., M.H., X.-Y.M., Z.Z., P.J., X.L., and M.C. analyzed data; B.L. and M.C. wrote the paper.

We gratefully acknowledge financial support from National Institute Health Grants R21DC008996 (M.C.) and S10RR027411 (M.C.). The computations were supported by the Teragrid Supercomputing grant (TG-MCB090019) and the Center for High Performance Computing and Institute of Structural Biology and Drug Discovery at Virginia Commonwealth University. We are grateful to Profs. Diomedes E. Logothetis and John A. DeSimone for their critical reading of the manuscript.

The authors declare no competing financial interests.

X. Li's present address: AmeriPath Northeast, 1 Greenwich Place, Shelton, CT 06484

Correspondence should be addressed to Meng Cui, Department of Physiology and Biophysics, Virginia Commonwealth University, 1220 East Broad Street, P.0. Box 980551, Richmond, VA 23298. E-mail: mcui@vcu.edu.

DOI:10.1523/JNEUROSCI.0791-11.2011

Copyright $\odot 2011$ the authors $\quad 0270-6474 / 11 / 3111070-07 \$ 15.00 / 0$
}

binding sites in the heterodimeric receptor (Jiang et al., 2004, 2005a,b; Xu et al., 2004; Nie et al., 2005; Winnig et al., 2005, 2007; Cui et al., 2006). For example, the artificial sweeteners aspartame and neotame bind to the VFTM domain of the human Tas1R2, whereas cyclamate, neohesperidin dihydrochalcone, and the sweet taste inhibitor lactisole interact with the TMD of the human Tas1R3 (Xu et al., 2004; Jiang et al., 2005c; Winnig et al., 2007; Zhang et al., 2010). We developed a homology model of the VFTM of hTas1R2, and predicted the potential binding site of the sweet taste receptor for aspartame and neotame (Cui et al., 2006, 2008). Behavioral studies have shown that the synthetic sweeteners aspartame and neotame and sweet-tasting proteins can be perceived by human, apes, and Old World monkeys but not by New World monkeys and rodents (Hellekant and Danilova, 1996; Jiang et al., 2004; Li et al., 2009). The molecular basis of species differences in taste sensitivity toward these sweeteners remains an open question. Furthermore, up to the present time, the Tas1R2/3 receptors from humans, mice, and rats but not monkeys have been functionally characterized (Nelson et al., 2001; Cui et al., 2006; Zhang et al., 2010). Therefore, to uncover the molecular determinants of species-dependent sweet taste perception of these sweeteners, it is important to investigate the nature of their interactions with the sweet taste receptors from closely related species, e.g., between the human and New World monkey species.

In this study, we heterogeneously expressed and functionally assessed the heteromeric receptor of Tas1R2 and Tas1R3 from squirrel monkey (Saimiri sciureus, named smTas1R2 and smTas1R3, respectively), which belongs to the genus Saimiri of New World monkeys. Using receptor chimeras between human 
and squirrel monkey, mutagenesis, and molecular modeling, we demonstrate that human/squirrel monkey species-dependent sweet taste differences are determined by a combination of a few specific interaction sites, which promise to help us understand the evolution of mammalian sweet perception at a molecular level.

\section{Materials and Methods}

Constructs. The coding nucleotide acid sequence and deduced amino acid sequence of squirrel monkey (of either sex) Tas1R2 and Tas1R3 were as described previously (Li et al., 2009, 2011). To generate the smTas1R2 expression construct, BamHI and NotI sites were introduced at the $5^{\prime}$ and $3^{\prime}$ ends of the full-length coding sequence, respectively, double digested, and then ligated into the expression vector pcDNA3.1. To obtain the smTas1R3 construct, the full protein-coding region of smTas $1 \mathrm{R} 3$ was synthesized from the GenScript Biology CRO. An EcoRI site was then introduced at the $5^{\prime}$ end before the start codon, and a NotI site was introduced at the $3^{\prime}$ end after the stop codon. The smTas1R3 gene was double restriction digested with EcoRI and NotI and then ligated into the expression vector pcDNA3.1. Human Tas1R2/3 (hTas1R2/3) expression constructs were generated in pcDNA3.1 vectors. The G $\alpha 16$-gust 44 clone was as described previously (Ueda et al., 2003).

Human/squirrel monkey (of either sex) Tas1R2 chimeras were constructed by using Sfil sites at human Tas1R2 amino acid 572 and squirrel monkey Tas1R2 amino acid 567. Human and squirrel monkey Tas1R2 single or multiple site mutations were made by standard PCR-based mutagenesis strategy (Stratagene). All the constructs were confirmed by DNA sequencing.

Functional assay. HEK293E cells were cultured at $37^{\circ} \mathrm{C}$ in Opti-MEM (Invitrogen), supplemented with 5\% fetal bovine serum (FBS). Cells for calcium imaging were seeded onto 96-well plates at a density of 50,000 per well and were cotransfected using Lipofectamine 2000 (Invitrogen) with plasmid DNAs encoding Tas1Rs (0.06 $\mu$ g per well) and G $\alpha 16$ gust44 $(0.06 \mu \mathrm{g})$. After $20 \mathrm{~h}$, the medium was changed once, after an additional $24 \mathrm{~h}$, the cells were washed with HBSS supplemented with 20 mM HEPES (HBSSH), loaded with $50 \mu$ l of $3 \mu \mathrm{m}$ Fluo-4 AM (Invitrogen) in $\mathrm{HBSSH}$, incubated for $1 \mathrm{~h}$, and then washed three times with $\mathrm{HBSSH}$ and maintained in $50 \mu \mathrm{l}$ of HBSSH. The plates were then placed into a FlexStation 3 system (Molecular Devices) to monitor the fluorescence change (excitation, $488 \mathrm{~nm}$; emission, $525 \mathrm{~nm}$; cutoff, $515 \mathrm{~nm}$ ) after the addition of $50 \mu \mathrm{l}$ of HBSSH supplemented with $2 \times$ tastants. For each trace, a tastant was added $30 \mathrm{~s}$ after the start of the scan. Scanning was continued for the additional $150 \mathrm{~s}$, and data were collected every $2 \mathrm{~s}$. Unless noted, the concentrations of the compounds used were as follows: aspartame, $10 \mathrm{~mm}$; neotame, $1 \mathrm{~mm}$; sucralose, $2 \mathrm{~mm}$.

Data analysis. Calcium mobilization in response to tastants was quantified as the percentage of change (peak fluorescence - baseline fluorescence level, denoted as $\Delta F$ ) from its baseline fluorescence level (denoted as $F)$. Data are presented as means $\pm \mathrm{SE}$ of the $\Delta F / F$ value from three independent experiments. GraphPad Prism 5 software (GraphPad Software) was used for generation of bar graphs and curve fitting. Statistical significance was determined by a Student's $t$ test or one-way ANOVA with Tukey's multiple-comparison test.

Molecular modeling. The homology model of hTas1R2/hTas1R3 VFTM (closed-open/active form) was constructed with the MODELLER program (Sali and Blundell, 1993; Martí-Renom et al., 2000) using the mGluR1-VFTM crystal structure (PDB entry: 1EWK) (Kunishima et al., $2000)$ as the template. A multiple sequence alignment of the VFTMs of hTas1R2, hTas1R3, mouse Tas1R2 (mTas1R2), mouse Tas1R3 (mTas1R3), and mGluR1 was generated by ClustalW (Thompson et al., 1994), followed by minor manual adjustments in the nonhomologous regions. The model of the VFTMs was refined with the CHARMM program (Brooks et al., 2010) according to the following protocol: the model was surrounded with a $6 \AA$ water shell and minimized for 5000 steps of steepest descent (SD) with the VFTM fixed. This was followed by 5000 steps of SD minimization with the backbone of the VFTM fixed, 5000 steps of SD minimization without restraints, and 40,000 steps of adapted basis Newton-Raphson (ABNR) minimization or until the root mean square energy gradient of $0.05 \mathrm{kcal} \cdot \mathrm{mol}^{-1} \cdot \AA^{-1}$ has been achieved. The refined model then was evaluated by the Verify3D program (Bowie et al., 1991; Lüthy et al., 1992) with acceptable scores.

An automatic molecular docking program, AUTODOCK (Morris et al., 1998), was used for the docking studies. A grid map was generated for the hTas1R2 VFTM using CHNO (i.e., carbon, hydrogen, nitrogen, and oxygen) elements sampled on a uniform grid containing $87 \times 77 \times 75$ points, $0.375 \AA$ apart. The Lamarckian genetic algorithm was selected to identify the binding conformations of the ligands. One hundred docking simulations were performed for each of the ligands. The final docked aspartame and neotame configurations were selected based on docked binding energies and cluster analysis. The docked aspartame/neotamehTas1R2 complexes were then refined by CHARMM with a $6 \AA$ water shell using the same protocol as described above (Cui et al., 2006, 2008).

To identify the potential residues that could affect the speciesdependent sweet taste toward aspartame, we selected 31 residues within $6.5 \AA$ from aspartame based on the aspartame-receptor complex model. Based on the sequence alignment and model selection, four residues, S40, V66, I67, and D142, in humans and their corresponding residues T40, L66, S67, and E142 in squirrel monkeys are not conserved. We constructed hTas1R2:S40T/D142E double mutant by using the Sybyl program (Tripos). The mutated receptor was subjected to 200 steps of energy minimization, followed by 6 ps Molecular Dynamics (MD) simulations using Tripos force field with a distance-dependent dielectric constant (5r) to mimic the solvation effect. During the MD simulations, only the mutated residues and adjacent residues were allowed to move, while the rest of the receptor remained fixed. For comparison, simulations of wildtype hTas1R2-VFTM with and without aspartame present were also performed using the same computational protocol. The simulations were performed on a local Linux computer (Intel Core 2 Duo Processor).

\section{Results}

The extracellular domain of Tas1R2 contains critical regions regulating the species difference of sweet taste toward aspartame and neotame

It has been reported that the extracellular domain of human Tas1R2 determines the response to the artificial sweeteners aspartame, neotame, and sucralose (Xu et al., 2004; Cui et al., 2006; Zhang et al., 2010). We loaded HEK-293E cells plated in 96-well plates with the $\mathrm{Ca}^{2+}$-sensitive dye Fluo-4 AM and monitored intracellular $\mathrm{Ca}^{2+}$ concentration changes resulting from ligand binding to Tas1R2/Tas1R3 receptors by measuring fluorescence changes in a FlexStation 3 system (see Materials and Methods). As shown in Figure $1 A$, the squirrel monkey Tas1R2/3 receptor did not respond to aspartame and neotame compared with control (the receptor response to HBSSH solution buffer) but did respond to sucralose, D-tryptophan, and sucrose, consistent with previously reported behavioral data (Glaser et al., 1995; Nofre et al., 1996). The hTas1R2 and smTas1R3 mismatched receptor was able to respond to aspartame and neotame compared with the control, but the smTas1R2/hTas1R3 was not, in contrast with the response to sucralose, D-tryptophan, and sucrose, which remained intact or reduced. These results suggested that specific residues in hTas1R2 are required for the receptor's response to aspartame and neotame. Figure $1 B$ shows a schematic representation of the heterodimer of Tas1R2 and Tas1R3 sweet taste receptor. To test whether the hTas1R2 critical region for sweetener sensitivity resided in the extracellular domain, chimeras between the human and squirrel monkey Tas1R2 receptors were constructed by swapping the N-terminal domains, where aspartame and neotame have been reported to act. The $\mathrm{h}(1-572)$ smTas1R2 contained the N-terminal domain (1-572 residues) of hTas1R2 and the transmembrane and intracellular domains (573-833) of smTas1R2, while the $\operatorname{sm}(1-567) \mathrm{hTas} 1 \mathrm{R} 2$ contained the N-terminal domain (1-567 residues) of smTas1R2 and the transmembrane and intracellular domains (568-839) of hTas1R2. The 

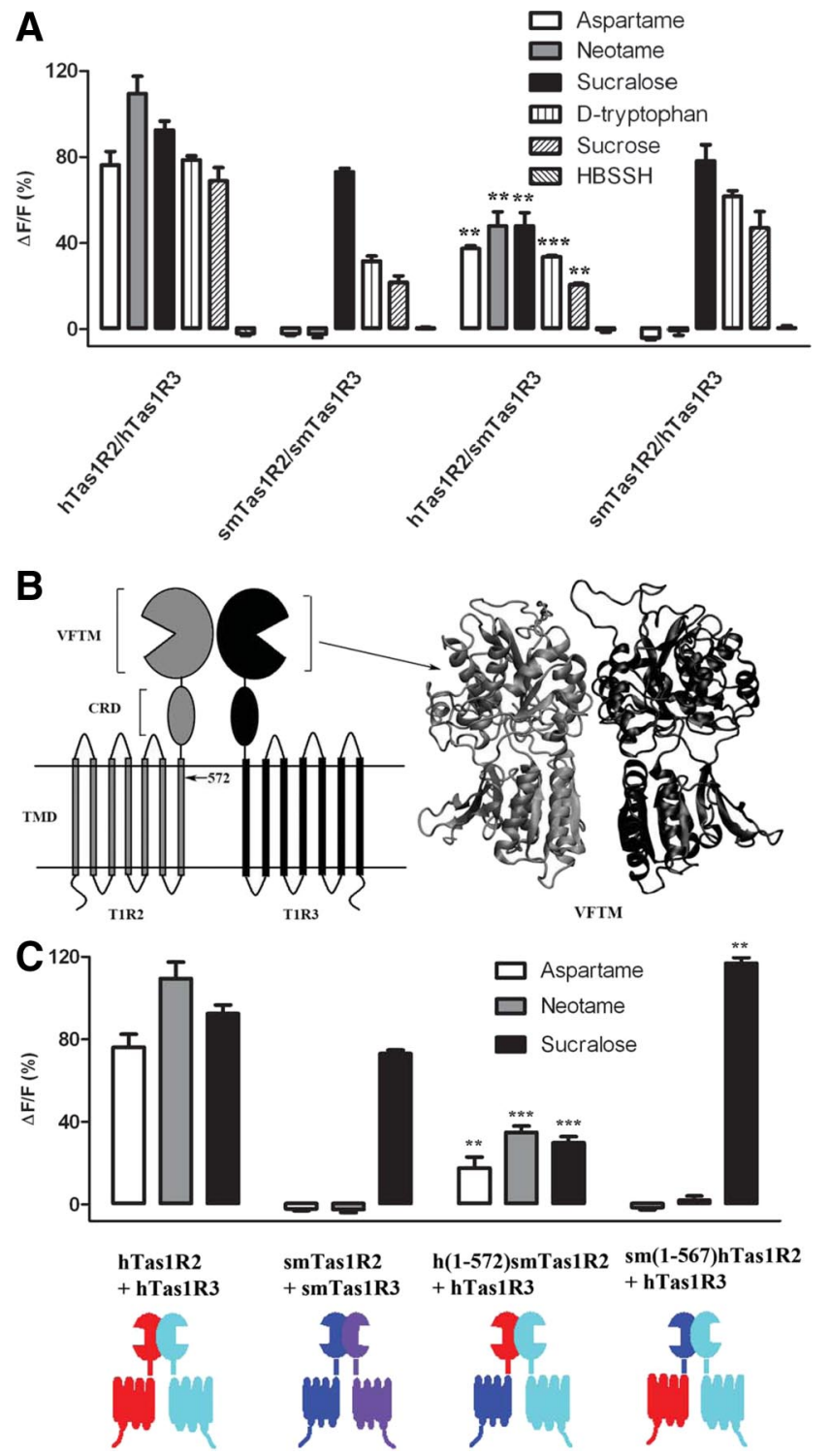

Figure 1. Mapping the binding region ofhTas 1R2 for aspartameand neotame. $A$, Human, squirrel monkey, or human + squirrel monkey mismatched sweet taste receptors with $G \alpha 16$-gust44 were cotransfected and expressed in HEK293E cells, and $\mathrm{Ca}^{2+}$ mobilization in response to the sweeteners aspartame (10 mM), neotame (1 mM), sucralose ( $2 \mathrm{~mm})$, o-tryptophan (5 mM), and sucrose (150 mM) and HBSSH buffer were measured. The calcium response signals were normalized to the maximum response of $\mathrm{hTas} 1 \mathrm{R} 2 / \mathrm{hTas} 1 \mathrm{R} 3$ receptor to sucralose $(2 \mathrm{~mm})$. Fis the baseline level of fluorescence, and $\Delta$ F is the change in fluorescence from the baseline level (peak - baseline). Data are expressed as the mean $\pm S E M$ of the $\Delta F / F$ values from three independent experiments. The asterisk indicates significant differences tested by unpaired Student's $t$ test compared with hTas1R2/hTas1R3 receptors $\left({ }^{* *} p<0.01 ; *{ }^{* *} p<0.001\right) . B$, Schematic representation of the heterodimer of Tas1R2 and Tas1R3. The arrow points to the site 572 , which is the Sfil enzyme cleavage site for both human and squirrel monkey Tas1R2 monomers. The VFTM of hTas1R2/hTas1R3 homology model is represented as a newcartoon color coded by gray and black for hTas $1 R 2$ and hTas $1 R 3$, respectively, by using VMD program (Hymphrey etal., 1996). C, The responses of hTas 1R2/hTas1R3and smTas1R2/smTas1R3 are the same as those shown in $A$, which is for comparison with human/squirrel monkey chimeras' [h(1572)smTas1R2 is a chimera of hTas1R2 (1-572) + smTas1R2 (573-833), and sm(1-567)hTas1R2 is a chimera of smTas1R2 (1--567) + hTas1R2 (568-839)] responses to the sweeteners aspartame (10 $\mathrm{mm})$, neotame (1 mM), and sucralose ( $2 \mathrm{~mm})$. The asterisks indicate significant differences tested by unpaired Student's $t$ test $\left({ }^{*} p<0.05 ;{ }^{* *} p<0.01 ;{ }^{* * *} p<0.001\right)$ compared with the hTas1R2/ hTas1R3 receptors. Diagram color coding: red, hTas1R2; cyan, hTas1R3; blue, smTas1R2; purple, smTas1R3.

$\operatorname{sm}(1-567) \mathrm{hTas} 1 \mathrm{R} 2$ chimera showed no responses to aspartame and neotame, when coexpressed with hTas1R3. Conversely, the $\mathrm{h}(1-572)$ smTas1R2 chimera showed sensitivity to aspartame and neotame, when paired with hTas1R3, albeit exhibiting signifi- cantly reduced responses (Fig. 1C). Our results are consistent with the report by Xu et al. (2004), wherein the Tas1R2 chimera that contains the N-terminal domain of human Tas1R2 paired with human TasT1R3 is able to respond to aspartame and neotame (the response is reduced compared to that of hTas1R2/ 3 ), whereas the reversed Tas1R2 chimera containing the $\mathrm{N}$-terminal domain of rat Tas1R2 is not. These results confirm that the extracellular domain of human Tas1R2 contains critical molecular determinants for activation by these two sweeteners.

The hTas1R2/smTas1R3 receptor shows reduced responses toward the sweeteners sucralose, aspartame, D-tryptophan, and sucrose (Fig. 1A) compared to the wild-type human receptor. Furthermore, the $\mathrm{h}(1-572)$ smTas1R2 chimera + hTas1R3 receptor shows significant reduction of the response toward aspartame, neotame, and sucralose, while sm(1-567)hTas1R2 chimera Tas1R3 + hTas1R3 receptor shows significant increase of the response toward sucralose (Fig. 1C). The response changes of these mismatched receptors could be attributed to a number of factors, such as coupling and cross talk between two protomers, and efficiency of coupling of the heteromer to the downstream signaling. We also noted that the mouse Tas1R2 and human Tas1R3 mismatched receptor was nonfunctional, showing no response to any sweeteners tested (Jiang et al., 2004). A reduction of the response was also observed for human and rat Tas1R2/ Tas1R3 chimera mismatched receptors to sucrose (Xu et al., 2004). Although we conclude the human Tas1R2 contains the critical regions regulating the species difference of sweet taste toward aspartame and neotame, we cannot completely rule out the potential role of hTas1R3, which could have some cooperative effects in the regulation of the species-dependent sweet taste.

\section{Identification of the human residues responsible for the} species-dependent sensitivity toward aspartame and neotame We proceeded to use human/squirrel monkey chimeras to further map the interaction sites in the receptor for the aspartame and neotame artificial sweeteners. BLAST analysis (http://www. ncbi.nlm.nih.gov/) revealed an $89 \%$ overall identity between the human Tas1R2 (accession no. Q8TE23) and the squirrel monkey Tas1R2 (A3QP08) sequences (Fig. 2A), compared to a 78\% identity between the human and mouse Tas1R2 (Q925I4) sequences. We focused on four residue differences between the human and squirrel monkey sequences that we previously predicted to be located near the binding pocket (Cui et al., 2006). These four residues, at positions $40,66,67$, and 142 , shown for the human Tas1R2 in Figure $2 B$, were probed for differences between the human and squirrel monkey Tas $1 \mathrm{R} 2$ receptors in response to the sweeteners aspartame and neotame. Figure $3 \mathrm{~A}$ shows that mutation of all four residues, hTas1R2:S40T/V66L/I67S/D142E, greatly impaired the ability of the human Tas1R2 to respond to aspartame and neotame. Similar results were obtained with the triple mutant hTas1R2:S40T/I67S/D142E. We proceeded to investigate the effect of mutation of each of these three residues (S40, I67, and D142), which greatly impaired responsiveness to aspartame and neotame. The hTIR2:I67S mutant exhibited a mild phenotype on the response to aspartame and slightly reduced response to neotame. Mutant hTas1R2:S40T abolished the receptor's response to aspartame, but did not affect significantly the response to neotame (interestingly the response to sucralose was also significantly reduced by this mutant). Mutant hTas1R2: D142E almost abolished the receptor's response to aspartame and significantly decreased the response to neotame. These results suggested that responses to aspartame versus neotame were differentially influenced by mutations at positions 40 and 142 . 


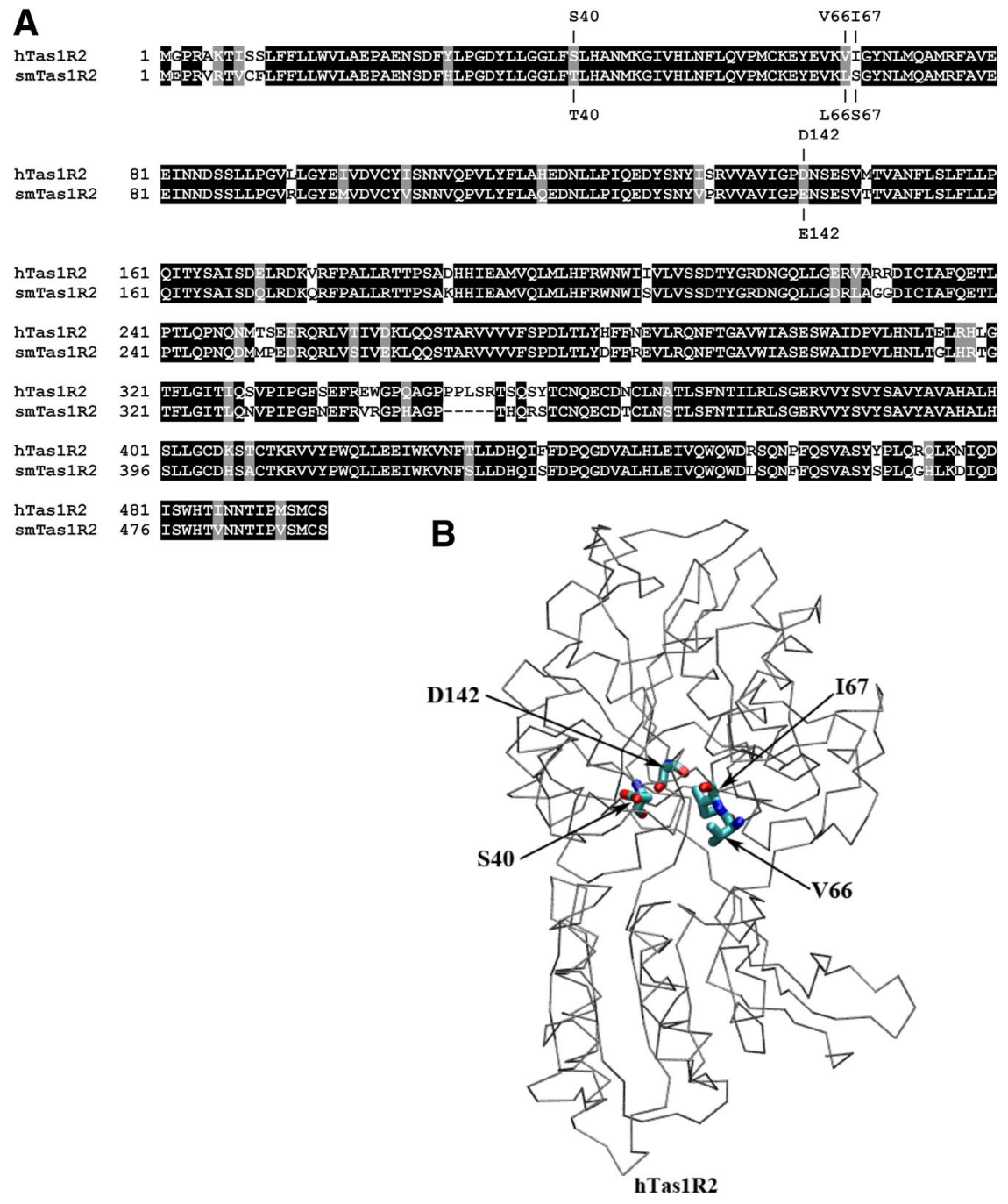

Figure 2. Predicted critical residues determine the species dependence of sweet taste. $\boldsymbol{A}$, Sequence alignment between the VFTMs of $h$ Tas 1R2 and smTas 1R2. Conserved residues are shaded by black, and semiconservative residues are shaded by gray. The model-predicted four critical amino acids involved in human and squirrel monkey sweet taste selectivity toward aspartame and neotame are marked above and below the sequences. $\boldsymbol{B}$, The location of predicted four key residues, $S 40, \mathrm{~V} 66,167$, and D142, in the VFTM of hTas1R2 homology model. The receptor is represented as $C \alpha$ trace color coded by gray, and the key residues are represented as a licorice model color coded by atom type.

We next tested response levels to double mutants. hTas1R2: S40T/D142E abolished the sweet response to aspartame (similar to the S140T and D142E effects) and significantly reduced the response to neotame (similar to the D142E effect). The I67S/D142E double mutant exhibited a similar response, namely an abolition of the aspartame response and significant reduction of the neotame response. This double mutant also exhibited an additive effect of I67S and D142E on neotame, and significantly reduced the response compared to D142E. In contrast, although the S40T/I67S double mutant showed impaired responses to all sweeteners, its response to aspartame was greater than multiple mutants that contained the D142E mutant.

Dose-response experiments illustrated that the responses of the mutants containing D142E to aspartame were abolished (Fig. 3B). Relative to the wild-type hTas1R2 neotame responses $\left(\mathrm{EC}_{50}=4.5 \times\right.$ $10^{-8} \mathrm{M}$ ), the triple mutant exhibited the lowest response (hTas1R2: S40T/I67S/D142E, $\mathrm{EC}_{50}=1.2 \times 10^{-2} \mathrm{M}$ ) compared to those of the double mutants (hTas1R2:S40T/D142E, $\mathrm{EC}_{50}=1.4 \times 10^{-3} \mathrm{M}$, and hTas1R2:I67S/D142E, $\left.\mathrm{EC}_{50}=4.7 \times 10^{-3} \mathrm{M}\right)($ Fig. $3 C$ ). These results suggest that residues $\mathrm{S} 40, \mathrm{I} 67$, and D142 are likely to be specific residues, which critically determine differences in species-dependent sweet taste response between human and squirrel monkey to the artificial sweeteners aspartame and neotame.

Engineering the squirrel monkey sweet receptor to respond to aspartame and neotame

If the identified residues are critical determinants of differences between the human and squirrel monkey receptor responses to sweeteners, then they could be sufficient to recover the sweet response of squirrel monkey to aspartame and neotame. We tested the function of humanized smTas1R2 toward these sweeteners (Fig. 3D). Both the quadruple (smTas1R2:T40S/L66V/S67I/E142D) and the triple (smTas1R2:T40S/S67I/E142D) mutant showed significant responses to neotame and aspartame compared to the control squirrel monkey receptor. The smTas1R2:T40S and S67I single mutants were not able to recover a sweet response of the receptor toward aspartame and neotame, while the smTas1R2:E142D single mutant recovered $\sim 30 \%$ of the receptor's response to aspartame compared to human Tas1R2/3 receptor. The smTas1R2:T40S/E142D double mutant showed enhanced responses toward these two sweeteners, but the smTas1R2:T40S/ S67I double mutant exhibited no response to aspartame and neotame. These data suggest that the residue D142 in hTas1R2 is crucial for sensitivity to aspartame and neotame and are consistent with the results of Figure $3 A-C$. Finally, the smTas1R2:S67I/E142D double mutant reproduced the higher neotame to aspartame sensitivity of the human receptor, suggesting that the human residue I67 may be responsible for the binding affinity difference between aspartame and neotame. Dose-response curves clearly showed that smTas1R2:T40S/E142D had the maximal recovered response to aspartame $\left[\mathrm{EC}_{50}=1.4 \times 10^{-3} \mathrm{M}\right.$, vs hTas1R2 $\left(\mathrm{EC}_{50}=\right.$ $\left.5.0 \times 10^{-3} \mathrm{M}\right)$ ], and smTas1R2:T40S/S67I/E142D had a significantly recovered response to neotame $\left[\mathrm{EC}_{50}=2.8 \times 10^{-4} \mathrm{M}\right.$, vs hTas1R2 $\left.\left(\mathrm{EC}_{50}=1.4 \times 10^{-5} \mathrm{M}\right)\right]$ (Fig. $\left.3 E, F\right)$. These two mutants showed stronger responses to the sweeteners than did the mutant smTas1R2:S67I/E142D, suggesting that residue S40 in hTas1R2 plays an auxiliary role to residue D142. Furthermore, the quadruple mutant smTas1R2:T40S/L66V/S67I/E142D showed only partial response of the maximal recovered response, suggesting that humanized residue V66 may have adverse effects in regaining the response. All the human and squirrel monkey mutants showed a significant level of response to the control, sucralose, suggesting that the gain or loss of response toward aspartame and neotame was not due to cell surface expression, misfolding, or targeting of the mutated proteins. These results demonstrate that specific combinations of a relatively few number of residues of the recep- 
$\mathbf{A}^{12}$
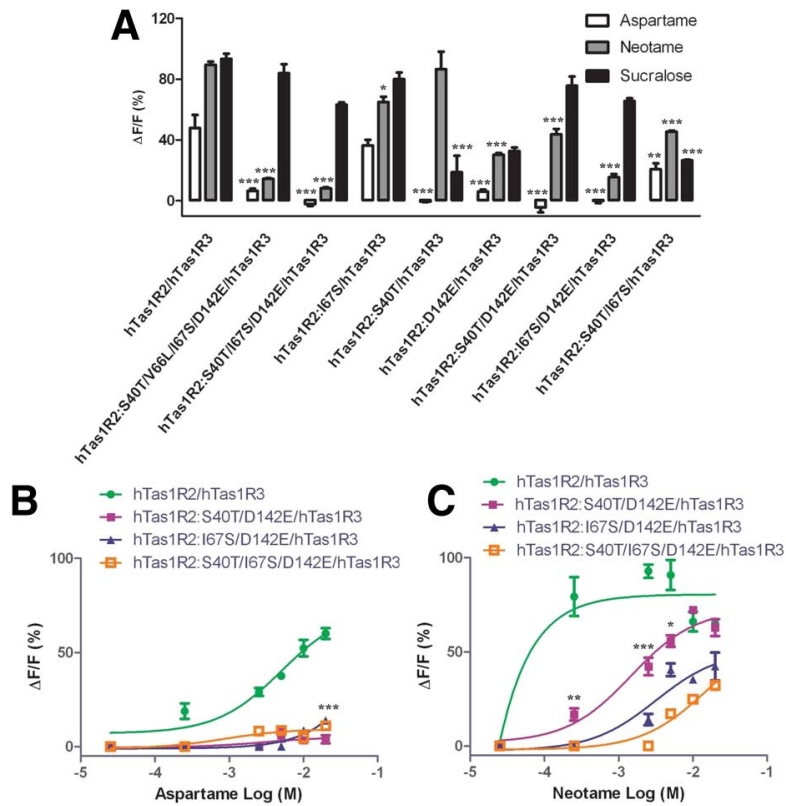

D $\left.{ }^{120}\right] \quad \square$ Aspartame
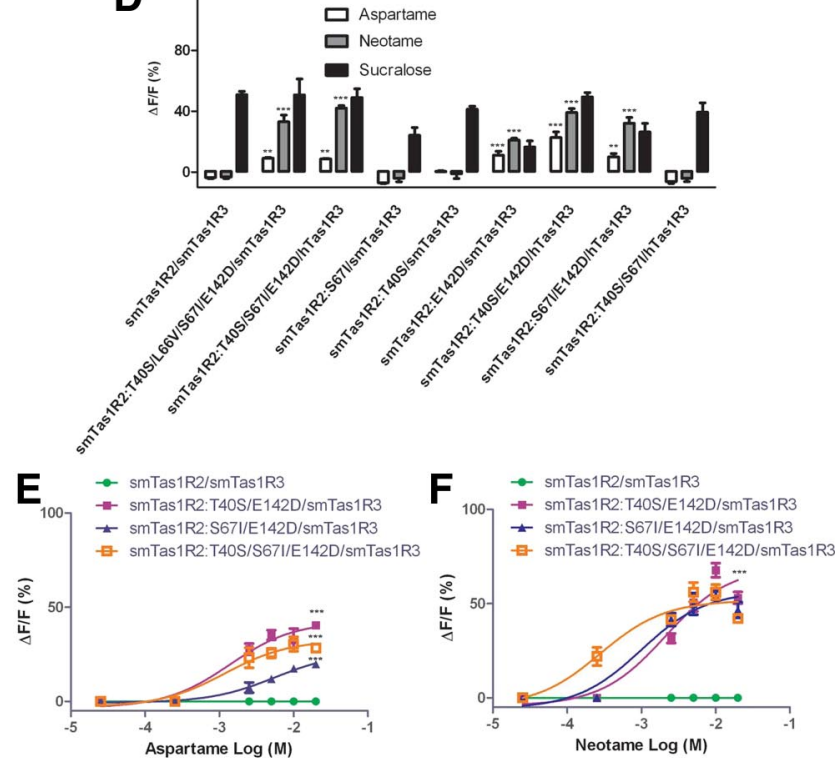

Figure 3. Evaluation of single or multiple point mutation affecting the human/squirrel monkey sweet taste receptors responses to sweeteners. $A$, HEK293E cells transiently transfected with human [hTas1R2/hTas1R3 (control)] and mutated hTas1R2 receptors along with $\mathrm{G}_{\alpha 16^{-}}$ gust44. The responses of the cells to aspartame $(10 \mathrm{~mm})$, neotame $(1 \mathrm{~mm})$, and sucralose $(2 \mathrm{~mm})$ were assayed by calcium mobilization. The calcium response signals were normalized to the maximum response of hTas1R2/hTas1R3 receptor to sucralose $(2 \mathrm{~mm})$. Values represent the mean \pm SEM of three independent experiments. The asterisk indicates significant differences tested by one-way ANOVA with Tukey's multiple-comparison test $\left({ }^{*} p<0.05\right.$; ${ }^{* *} p<0.01$; $\left.{ }^{* * *} p<0.001\right)$ compared with the hTas $1 R 2 / h T a s 1 R 3$ receptors. $B, C$, Dose-response of human and selected mutated human sweet taste receptors toward aspartame $(\boldsymbol{B})$ and toward neotame (C). The responses of the cells to concentrations of the sweeteners aspartame and neotame were assayed by calcium mobilization. The calcium response signals were normalized to the maximum response of $\mathrm{hTas} 1 \mathrm{R} 2 / \mathrm{hTas} 1 \mathrm{R} 3$ receptor to neotame. Mutants hTas1R2:S40T/ D142E + hTas1R3, hTas1R2:167S/D142E + hTas1R3, and hTas1R2:S40T/167S/D142E + hTas1R3 showed no responses to aspartame versus good response of hTas $1 \mathrm{R2}+\mathrm{hTas} 1 \mathrm{R3}\left(\mathrm{EC}_{50}=5.0 \times\right.$ $10^{-3} \mathrm{M}, \mathrm{B}: 7.074$, T: 73.32). Mutants hTas1R2:S40T/167S/D142E+hTas1R3 ( $\mathrm{EC}_{50}=1.2 \times$ $\left.10^{-2} \mathrm{M}, \mathrm{B}:-1.486, \mathrm{~T}: 39.87\right)$, hTas1R2:S40T/D142E+hTas1R3 (EC $\mathrm{E}_{50}=1.4 \times 10^{-3} \mathrm{M}, \mathrm{B}$ : 1.387, T: 61.12), and hTas1R2:167S/D142E + hTas1R3 $\left(\mathrm{EC}_{50}=4.7 \times 10^{-3} \mathrm{M}, \mathrm{B}:-1.772\right.$, T: 51.81) showed weaker responses to neotame versus good response of hTas1R2 $+\mathrm{hTas} 1 \mathrm{R3}\left(\mathrm{EC}_{50}=\right.$ $\left.\left.1.4 \times 10^{-5} \mathrm{M}, \mathrm{B}:-165.1, \mathrm{~T}: 92.82\right)\right)$. The asterisks indicate significant differences tested by unpaired Student's t test $\left({ }^{*} p<0.05 ;{ }^{* *} p<0.01 ;{ }^{* * *} p<0.001\right)$ compared with the hTas 1R2/ $\mathrm{hTas} 1 \mathrm{R} 3$ receptors. D, HEK293E cells transiently transfected with squirrel monkey [smTas1R2/ tor determine the species-selective sweet taste for the two artificial sweeteners.

\section{Steric effects of residues determine the species-dependent sweet taste difference between human and squirrel monkey toward aspartame and neotame}

Previously, we identified the potential binding sites in human sweet taste receptor for artificial sweeteners aspartame and neotame by using a combination of molecular modeling and experimental approaches (Jiang et al., 2005a; Cui et al., 2006, 2008). Here, we constructed a double mutant model of hTas1R2:S40T/ D142E based on our previous developed homology model of hTas1R2-VFTM. We compared short-time MD simulations on the site of the mutations and the surrounding area for both the mutant receptor and the wild-type receptor with and without aspartame present (Fig. 4). Residues T40 and E142 in the squirrel monkey receptor possess larger side chains than residues S40 and D142 in the human receptor. Our simulations predicted that these doubly mutated residues T40 and E142 can alter the conformation of the side chain of residue Y103, and therefore indirectly interact with the residue $\mathrm{N} 143$. The conformational changes of the side chain of residue N143 reduce the size of the binding site in the receptor for aspartame and lead to no response toward the sweetener. Figure $4 B$ shows that there is an overlap between the phenol ring of aspartame and the side chain of residue N143 in the mutated receptor. These results show how the steric effects of these two residues work together to determine the species-dependent sweet taste between the human and squirrel monkey receptors toward aspartame and neotame.

\section{Discussion}

In this paper, we functionally expressed the newly cloned squirrel monkey sweet taste receptor Tas1R2 and Tas1R3 in HEK293E cells. To our knowledge, this is the first characterized sweet taste receptor of a New World monkey species in an in vitro heterologous expression system ( $\mathrm{Li}$ et al., 2009). Previously, the role of hTas1R3 could not be evaluated based on mismatching with mouse Tas1R2 since the mismatched receptors were not functional (Jiang et al., 2004). Our functionally expressed smTas1R2+ hTas1R3 receptor assay overcame this problem, providing a useful tool to map the

smTas $1 R 3$ (control)] and mutated smTas $1 R 2$ receptors along with $\mathrm{G}_{16}$-gust 44 . The responses of the cells to aspartame ( $10 \mathrm{~mm})$, neotame $(1 \mathrm{~mm})$, and sucralose $(2 \mathrm{~mm})$ were assayed by calcium mobilization. The calcium response signals were normalized to the maximum response of $\mathrm{hTas} 1 \mathrm{R} 2 / \mathrm{hTas} 1 \mathrm{R} 3$ receptor to sucralose $(2 \mathrm{~mm})$. Values represent the mean \pm SEM of three independent experiments. The asterisk indicates significant differences tested by one-way ANOVA with Tukey's multiplecomparison test $\left({ }^{* *} p<0.01 ;{ }^{* *} p<0.001\right)$ compared with the smTas 1R2/smTas1R3 receptors. $\boldsymbol{E}$, $\boldsymbol{F}$, Dose-response of squirrel monkey, and selected mutated squirrel monkey sweet taste receptor toward aspartame $(\boldsymbol{E})$ and toward neotame $(\boldsymbol{F})$. The responses of the cells to concentrations of the sweeteners aspartame and neotame were assayed by calcium mobilization. The calcium response signals were normalized to the maximum response of hTas1R2/hTas1R3 receptor to neotame $(\boldsymbol{B})$. Mutants smTas1R2:T40S/E142D + smTas1R3 ( $\left.\mathrm{EC}_{50}=1.4 \times 10^{-3} \mathrm{M}, \mathrm{B}:-3.314, \mathrm{~T}: 42.4\right)$, sm Tas1R2:S67//E142D + smTas1R3 (EC $\left.{ }_{50}=5.6 \times 10^{-3} \mathrm{M}, \mathrm{B}:-0.6682, \mathrm{~T}: 26.17\right)$, and smTas1R2: $\mathrm{T} 40 \mathrm{~S} / \mathrm{S} 67 \mathrm{I} / \mathrm{E} 142 \mathrm{D}+\operatorname{smTas} 1 \mathrm{R3}\left(\mathrm{EC}_{50}=1.2 \times 10^{-3} \mathrm{M}, \mathrm{B}:-2.882, \mathrm{~T}: 32.61\right)$ showed good response to aspartame versus no response of smTas1R2 + smTas1R3. Mutants smTas1R2

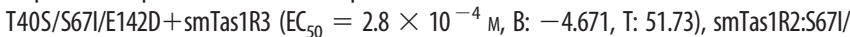
E142D $+\operatorname{smTas} 1 \mathrm{R3}\left(\mathrm{EC}_{50}=1.0 \times 10^{-3} \mathrm{M}, \mathrm{B}:-5.754, \mathrm{~T}: 56.59\right)$, and smTas1R2:T40S/ $\mathrm{E} 142 \mathrm{D}+\operatorname{smTas} 1 \mathrm{R3}\left(\mathrm{EC}_{50}=2.1 \times 10^{-3} \mathrm{M},-4.24, \mathrm{~T}: 69.46\right)$ showed good responses to neotame versus no response of smTas1R2 + smTas1R3. The asterisks indicate significant differences tested by unpaired Student'sttest $\left.{ }^{* * *} p<0.001\right)$ compared with the smTas1R2/smTas1R3 receptors. Data ( $\boldsymbol{B}$, $\boldsymbol{C}, \boldsymbol{E}, \boldsymbol{F})$ were fitted with sigmoid dose-response curves $[Y=$ Bottom $+($ Top - Bottom $) /(1+$ $\left.\left.10^{\log E_{5_{0}}-X}\right)\right]$ using GraphPad Prism software, and expressed as the mean \pm SEM of the $\Delta F / F$ values from three independent experiments. 


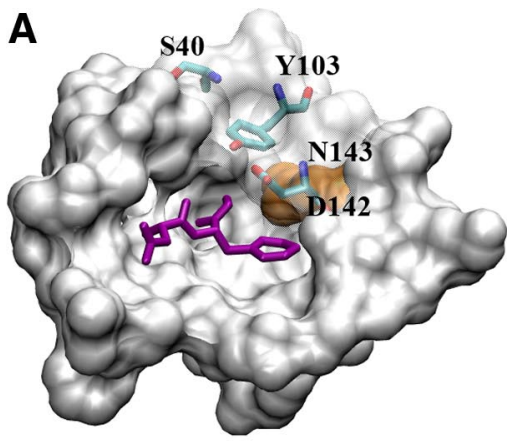

hTas1R2 + Aspartame

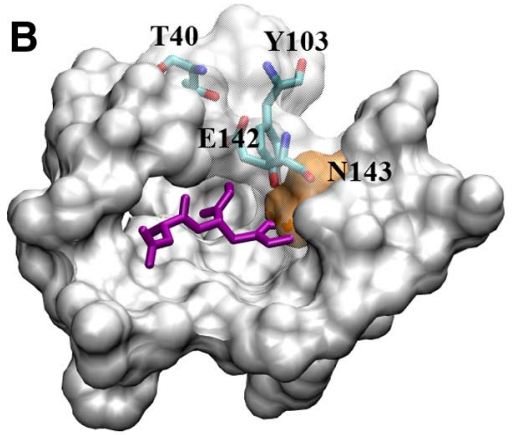

hTas1R2:S40T/D142E +Aspartame

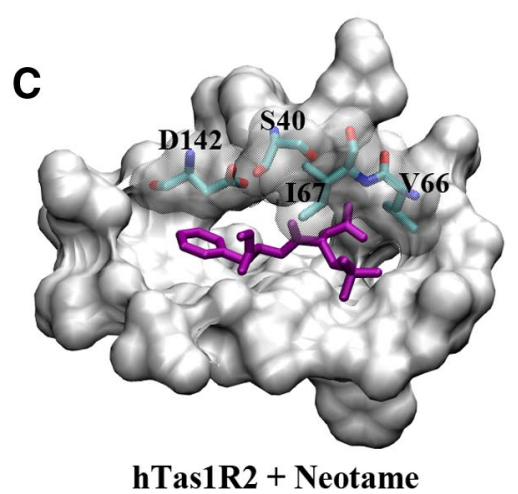

Figure 4. The refined docking complex structures of the VFTM domain of hTas1R2. $\boldsymbol{A}, \boldsymbol{B}$, Aspartame is docked within the binding pockets of $h$ Tas 1 R2 wild-type receptor $(\boldsymbol{A})$ and mutated receptor hTas1R2:S40T:D142E (B). Only the binding pocket of hTas1R2 and the mutant with aspartame are shown. The pocket residues are illustrated by a molecular surface colored with silver except for the residue N143 (in orange). Aspartame is rendered as a stick model and colored by purple. Five critical residues for the human/squirrel monkey recognition (S40, T40, Y104, D142, and E142) toward aspartame are rendered as stick models and colored by atom element. C, Neotame is docked within the binding pocket of hTas1R2 wild-type receptor. The pocket residues are illustrated by a molecular surface colored with silver. Neotame is rendered as a stick model and colored by purple. Four residues involved in the human/squirrel monkey recognition $(540, D 142, \mathrm{~V} 66$, and 167$)$ toward neotame are rendered as stick models and colored by atom type.

interaction sites of hTas1R3 in future studies. We then used molecular modeling and docking, mutagenesis, and functional studies to investigate molecular determinants of the species-dependent sweet taste between humans and squirrel monkey. Aspartame (L- $\alpha-$ aspartyl-L-phenylalanine methyl ester) is a dipeptide sweetener with 200 times the sweet potency of sucrose (Meguro et al., 2000). In our model, the main interactions between aspartame and the N-terminal extracellular domain of hTas1R2 can be classified as hydrogen bonds (S303, R383, and V384), salt bridges (D278 and D307), and hydrophobic interactions (Y103, Y215, and P277). Two water molecules are interactive with D142 and L279, respectively, to bridge aspartame with the binding sites (Cui et al., 2006). All these interactive residues are conserved between hTas1R2 and smTas1R2 except D142, which is replaced by Glu in smTas1R2. Xu et al. (2004) and Jiang et al. (2005a) have demonstrated that mutations of S144, E302, and D307 in hTas1R2 either abolished or had significant reductions on the responses of the human receptor to aspartame and neotame. Yet, these residues are all identical between the human and squirrel monkey Tas1R2s. Our molecular model prediction is consistent with the mutagenesis and functional expression data, which indicate that the residue D142 in hTas1R2 is the most important residue in determining the human/squirrel monkey sweet taste differences toward aspartame. Mutant hTas1R2:D142E abolishes the activity to aspartame and smTas1R2:E142D regains $\sim 30 \%$ of the response compared to the hTas $1 \mathrm{R} 2$ receptor. We also found that $\mathrm{S} 40$ in the hTas1R2 is a critical auxiliary residue since the double mutant smTas1R2:T40S/ E142D leads to response levels nearly equivalent to those of the human sweet taste receptor toward aspartame (Fig. $3 A, B, E$ ). The role of S40 is cooperative with D142 in facilitating the spatial orientation for agonist binding and stabilizing the closed conformation of the activated human receptor.

Neotame is an analog of aspartame that has an additional dimethylbutyl group and is $\sim 40$ times sweeter than aspartame (Garbow et al., 2001). One can speculate that additional residues may interact with this molecule. In our previous model, in addition to the aspartame-interacting residues, residues V64, V66, I67, V309, and L310 in hTas1R2 were identified to contribute to receptor activation by neotame through hydrophobic interactions (Cui et al., 2006, 2008). Among the five residues, V66 and I67 are replaced by L and S in smTas1R2, respectively. V66 and especially I67 in hTas1R2 seem to be engaged in neotame/aspartame taste specificity either by direct interaction or indirect modulation of the spatial orientation of the interactive residues. Indeed, according to our molecular modeling, residue I67 in hTas1R2 interacts with the dimethylbutyl group of neotame through hydrophobic interactions, while S67 in smTas1R2 is a hydrophilic residue and adverse to the binding affinity of the receptor for neotame (Fig. 4C). Our model is supported by the results that the double mutant hTas1R2:S40T/D142E reduced the response to neotame by half compared to the wild-type receptor, while the triple mutant hTas1R2:S40T/I67S/D142E almost completely abolished the response to neotame (Fig. 3A, C). Interestingly, the residue V66 in hTas1R2, which corresponds to the residue L66 in smTas1R2, has adverse effects for sweet response toward aspartame and neotame. Our results show that the quadruple mutant hTas1R2:S40T/V66L/I67S/D142E has a stronger response toward the sweeteners than the triple mutant hTas1R2:S40T/I67S/D142E as shown in Figure 3A. The larger residue Leu in this position could contribute stronger van der Waals interactions for both aspartame and neotame with the receptor (Fig. 4C). These data can be used as guidance for designing novel effective artificial sweet compounds.

In conclusion, using a combination of experimental and molecular modeling approaches, we depicted that human/squirrel monkey taste differences toward the synthetic sweeteners aspartame and neotame are determined by the spatial effect of a combination of a few interactive sites. The mechanism of interaction between sweet taste receptor and sweet compound has been under investigation for many years, and several models have been developed based on the structures of existing sweeteners to explain the physicochemical requirements of a sweet compound: the AH-B model of Shallenberger and Acree (1967), the AH, B, X 
model of Kier (1972), and the multipoint attachment theory model of Tinti and Nofre (1991). Bassoli et al. (2002) developed a general pseudoreceptor model for sweet compound using a training set of 24 compounds belonging to different families including sugars, peptides, and other intensive sweeteners, and showed very good correlation between the calculated binding free energies and relative sweetness. However, these models are all limited for the interplay and bear little resemblance to the coding logic of this repertoire. On the other hand, a hydrophobic binding site was suggested to be present as a requisite in the receptor of Old World primates to explain their sensitivity in sweet taste toward aspartame (Glaser et al., 1995). We have used extensive molecular modeling combined with experimental investigation to identify the binding pocket for aspartame in the receptor (Jiang et al., 2005a; Cui et al., 2006). Considering the higher sequence identity between human and squirrel monkey, it is logical that only a few residue differences located in the binging pocket could determine the species-dependent sweet taste toward some sweeteners. Extra residues of Tas1R2 could also be required to determine the human-mouse taste difference to aspartame and neotame, such as T171 (S168), V246 (T242), and E282 (D278), etc., in mouse Tas1R2 (hTas1R2), if we were to compare the human and mouse receptors. Our results strongly suggest that human/New World squirrel monkey species-dependent sweet taste toward the dipeptide sweeteners is determined by the steric interplay between sweeteners and cooperative combinations of a few essential residues in sweet taste receptors.

\section{References}

Bassoli A, Drew MG, Merlini L, Morini G (2002) General pseudoreceptor model for sweet compounds: a semiquantitative prediction of binding affinity for sweet-tasting molecules. J Med Chem 45:4402-4409.

Bowie JU, Lüthy R, Eisenberg D (1991) A method to identify protein sequences that fold into a known three-dimensional structure. Science 253:164-170.

Brooks BR, Bruccoleri RE, Olafson BD, States DJ, Swaminathan S, Karplus M (2010) CHARMM: a program for macromolecular energy, minimization, and dynamics calculations. J Comput Chem 4:187-217.

Cui M, Jiang P, Maillet E, Max M, Margolskee RF, Osman R (2006) The heterodimeric sweet taste receptor has multiple potential ligand binding sites. Curr Pharm Des 12:4591-4600.

Cui M, Jiang P, Maillet E, Max M, Margolskee RF, Osman R (2008) Molecular models of sweet taste receptors provide insights into function. In: Sweetness and sweeteners: biology, chemistry, and psychophysics (Weerasinghe DK, DuBois GE, eds), pp 117-132. Washington, DC: American Chemical Society.

Garbow JR, Likos JJ, Schroeder SA (2001) Structure, dynamics, and stability of beta-cyclodextrin inclusion complexes of aspartame and neotame. J Agric Food Chem 49:2053-2060.

Glaser D, Tinti JM, Nofre C (1995) Evolution of the sweetness receptor in primates. I. Why does alitame taste sweet in all prosimians and simians, and aspartame only in Old World simians? Chem Senses 20:573-584.

Hellekant G, Danilova V (1996) Species differences toward sweeteners. Food Chem 56:323-328.

Humphrey W, Dalke A, Schulten K (1996) VMD—visual molecular dynamics. J Mol Graphics 14:33-38.

Jiang P, Ji Q, Liu Z, Snyder LA, Benard LM, Margolskee RF, Max M (2004) The cysteine-rich region of T1R3 determines responses to intensely sweet proteins. J Biol Chem 279:45068-45075.

Jiang P, Cui M, Ji Q, Snyder L, Liu Z, Benard L, Margolskee RF, Osman R, Max
M (2005a) Molecular mechanisms of sweet receptor function. Chem Senses 30 [Suppl 1]:i17-i18.

Jiang P, Cui M, Zhao B, Liu Z, Snyder LA, Benard LM, Osman R, Margolskee RF, Max M (2005b) Lactisole interacts with the transmembrane domains of human T1R3 to inhibit sweet taste. J Biol Chem 280:15238-15246.

Jiang P, Cui M, Zhao B, Snyder LA, Benard LM, Osman R, Max M, Margolskee RF (2005c) Identification of the cyclamate interaction site within the transmembrane domain of the human sweet taste receptor subunit T1R3. J Biol Chem 280:34296-34305.

Kier LB (1972) A molecular theory of sweet taste. J Pharm Sci 61:1394-1397. Kunishima N, Shimada Y, Tsuji Y, Sato T, Yamamoto M, Kumasaka T, Nakanishi S, Jingami H, Morikawa K (2000) Structural basis of glutamate recognition by a dimeric metabotropic glutamate receptor. Nature 407:971-977.

Li X, Glaser D, Li W, Johnson WE, O’Brien SJ, Beauchamp GK, Brand JG (2009) Analyses of sweet receptor gene (Tas1r2) and preference for sweet stimuli in species of Carnivora. J Hered 100 [Suppl 1]:S90-S100.

Li X, Bachmanov AA, Maehashi K, Li W, Lim R, Brand JG, Beauchamp GK, Reed DR, Thai C, Floriano WB (2011) Sweet taste receptor gene variation and aspartame taste in primates and other species. Chem Senses 36:453-475.

Lüthy R, Bowie JU, Eisenberg D (1992) Assessment of protein models with three-dimensional profiles. Nature 356:83-85.

Martí-Renom MA, Stuart AC, Fiser A, Sánchez R, Melo F, Sali A (2000) Comparative protein structure modeling of genes and genomes. Annu Rev Biophys Biomol Struct 29:291-325.

Meguro T, Kashiwagi T, Satow Y (2000) Crystal structure of the lowhumidity form of aspartame sweetener. J Pept Res 56:97-104.

Morris GM, Goodsell DS, Halliday RS, Huey R, Hart WE, Belew RK, Olson AJ (1998) Automated docking using a Lamarckian genetic algorithm and an empirical binding free energy function. J Comput Chem 19:1639-1662.

Nelson G, Hoon MA, Chandrashekar J, Zhang Y, Ryba NJ, Zuker CS (2001) Mammalian sweet taste receptors. Cell 106:381-390.

Nie Y, Vigues S, Hobbs JR, Conn GL, Munger SD (2005) Distinct contributions of T1R2 and T1R3 taste receptor subunits to the detection of sweet stimuli. Curr Biol 15:1948-1952.

Nofre C, Tinti JM, Glaser D (1996) Evolution of the sweetness receptor in primates. II. Gustatory responses of non-human primates to nine compounds known to be sweet in man. Chem Senses 21:747-762.

Sali A, Blundell TL (1993) Comparative protein modelling by satisfaction of spatial restraints. J Mol Biol 234:779-815.

Shallenberger RS, Acree TE (1967) Molecular theory of sweet taste. Nature 216:480-482.

Thompson JD, Higgins DG, Gibson TJ (1994) CLUSTAL W: improving the sensitivity of progressive multiple sequence alignment through sequence weighting, position-specific gap penalties and weight matrix choice. Nucleic Acids Res 22:4673-4680.

Tinti JM, Nofre C (1991) Why does a sweetener taste sweet? A new model. In: Sweeteners: discovery, molecular design, and chemoreception (DuBois GE, ed), pp 206-213. Washington DC: American Chemical Society.

Ueda T, Ugawa S, Yamamura H, Imaizumi Y, Shimada S (2003) Functional interaction between T2R taste receptors and G-protein $\alpha$ subunits expressed in taste receptor cells. J Neurosci 23:7376-7380.

Winnig M, Bufe B, Meyerhof W (2005) Valine 738 and lysine 735 in the fifth transmembrane domain of rTas1r3 mediate insensitivity towards lactisole of the rat sweet taste receptor. BMC Neurosci 6:22.

Winnig M, Bufe B, Kratochwil NA, Slack JP, Meyerhof W (2007) The binding site for neohesperidin dihydrochalcone at the human sweet taste receptor. BMC Struct Biol 7:66.

Xu H, Staszewski L, Tang H, Adler E, Zoller M, Li X (2004) Different functional roles of T1R subunits in the heteromeric taste receptors. Proc Natl Acad Sci U S A 101:14258-14263.

Zhang F, Klebansky B, Fine RM, Liu H, Xu H, Servant G, Zoller M, Tachdjian C, Li X (2010) Molecular mechanism of the sweet taste enhancers. Proc Natl Acad Sci U S A 107:4752-4757. 